\title{
Bow Hunter's Syndrome in a Patient with a Right Hypoplastic Vertebral Artery and a Dynamically Compressible Left Vertebral Artery
}

\begin{abstract}
This is a case report of a 48-year-old man with multiple transient ischemic attacks and a known hypoplastic right vertebral artery (VA) who presented after a syncopal event while turning his head to the left. The objective of this study is to demonstrate the necessity of proper diagnosis and management of cerebrovascular pathology including imaging and surgical intervention in patients with known anatomical anomalies. This study was conducted at Massachusetts, United States of America. Our patient' s history was significant for a hypoplastic right VA and a stenotic segment of the right VA at the C3-C4 junction. There was also degeneration of the C3-C4 facet on the left, with osteophyte formation compressing the VA, and a fusion of the C2-C3 segment. Imaging demonstrated obliteration of the left VA flow with head rotation to the left and subsequent reconstitution of flow in the neutral position. After consultation, the patient decided to proceed with surgical management with an anterior cervical discectomy and fusion at the level of C3-C4. Symptoms of vertebrobasilar insufficiency including syncopal episodes resolved after treatment. VA anomalies, although uncommon, are important to understand. Our patient presented with an anomalous right VA, as well as severe degenerative changes to the $\mathrm{C} 2 / \mathrm{C} 3$ vertebrae that contributed to the development of Bow Hunter' $s$ syndrome. It is essential that proper monitoring and follow-up has to be carried out in patients with abnormal cerebral vasculature to minimize the occurrence of Bow Hunter's syndrome.
\end{abstract}

Keywords: Bow Hunter, hypoplastic, vertebrobasilar insufficiency

\section{Introduction}

This is a reported case of a patient turning his head to the left leading to Bow Hunter' $s$ Syndrome, which is also known as rotational occlusion of the vertebral artery (VA).

Bow Hunter' $s$ is a rare cause of temporary

posterior cerebral circulation ischemic symptoms secondary to compromi se

of VA. Symptoms include paroxysmal vertigo or nonspecific dizziness which

is possibly accompanied by nystagmus, tinnitus, syncope, blurred vision, nausea, or vomiting. ['] Occlusion is due to compression of a dominant VA by bony elements of the cervical spine triggered by turning head to one side or head tilting. ['] Etiology is related to large osteophytes or
David Z. Cai, Ryan P. Roach, John P. Weaver, Gerald T. McGillicuddy, Zachary M.

Mansell 4 , Jonathan P. Eskander5, Mark S. Eskander 4

Departments of Otolaryngology and

sAnesthesiology, Tulane

University, Louisiana,

¿Department of Orthopaedic Surgery, New York

University, New York, NY, Departments of ${ }_{2}$ Orthopedics and ${ }_{3}$ Neurosurgery, UMass Memorial Medical Center,

Massachusetts,

\section{Case Report}

Pathology often is a result of hypoplasia or stenosis of the nondominant VA. The symptoms are relieved by returning the head to the neutral position. Few cases result in infarction with permanent neurologic deficits.

A 48-year-old male was referred for evaluation to the outpatient clinic of our department for repetitive near syncopal episodes upon turning his head to the left. The This is an open access article distributed under the terms of the Creative Commons Attribution-NonCommercial-ShareAlike $\quad 3.0$ License, which allows others to remix, tweak, and build upon the work non-commercially, as long as the author is credited and the new creations are licensed under the identical terms.

For reprints contact: reprints@medknow.com patient had a known history of multiple transient ischemic attacks and a hypoplastic right VA. The patient noted that symptoms of "I ightheadedness and dimmed vision" resolved when his head returned to a central position.

Imaging with 
tomography angiography revealed a hypoplastic right $V A$, and a stenotic left VA segment at the C3-C4 junction. There was degeneration of the C3-C4 facet on the left, with osteophyte

formation compressing the left

VA.

A fusion between the $C 2$ and $C 3$ vertebral segments was also noted [Figures 1 and 2]. A magnetic resonance angiogram was used to evaluate VA function and brain perfusion. Appropriate cerebral perfusion with contributions from the posterior communicating vessels and left VA was noted with the patient' $s$ head in a neutral

How to cite this article: Cai DZ, Roach RP, Weaver JP, McGillicuddy GT, Mansell ZM, Eskander JP, et al. Bow hunter's syndrome in a patient with a right hypoplastic vertebral artery and a dynamically compressible left vertebral artery. Asian J Neurosurg 2018;13:133-5.
${ }_{4}$ Department of Orthopedics, Delaware

Orthopedic Associates, Newark, Delaware, USA

Address for correspondence:

Dr. Zachary M. Manse/l, 3401 Brandywine

Parkway, Suite 101, Wi lmington, DE

19803, USA.

E-mail: zackmansel/@gmail.com

Access this article online

Website: www.asianjns.org

DOI: $10.4103 / 1793-5482.181129$

Quick Response Code:

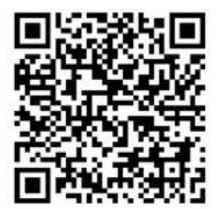


position [Figure 3]. Dynamic fluoroscopy of the left VA with head rotation to the left showed obliteration of left VA blood flow [Figure 4], and reconstitution of flow with the patient' $s$ head in the neutral position [Figure 5].

After full evaluation, the patient chose to undergo surgical treatment, and a traditional anterior cervical discectomy and fusion (ACDF) was performed at the level of C3-C4 resulting in immobilization of the unstable vertebral segment. At 4 months postoperative follow-up, the patient no longer experienced symptoms of vertebrobasilar insufficiency including syncopal episodes. Imaging demonstrates the ACDF [Figure $6]$.

\section{Discussion}

This case demonstrates symptomatic vertebrobasilar insufficiency as a result of occlusion of the left VA with physiologic head rotation known as Bow Hunter's syndrome. The level of VA occlusion at C3-C4 combined with the anatomical variant of a hypoplastic right VA makes the present case a rare presentation of Bow Hunter's syndrome. The anatomical relationship of the VA to neighboring structures accommodates compression or occlusion of the vessels with the segment of VA between
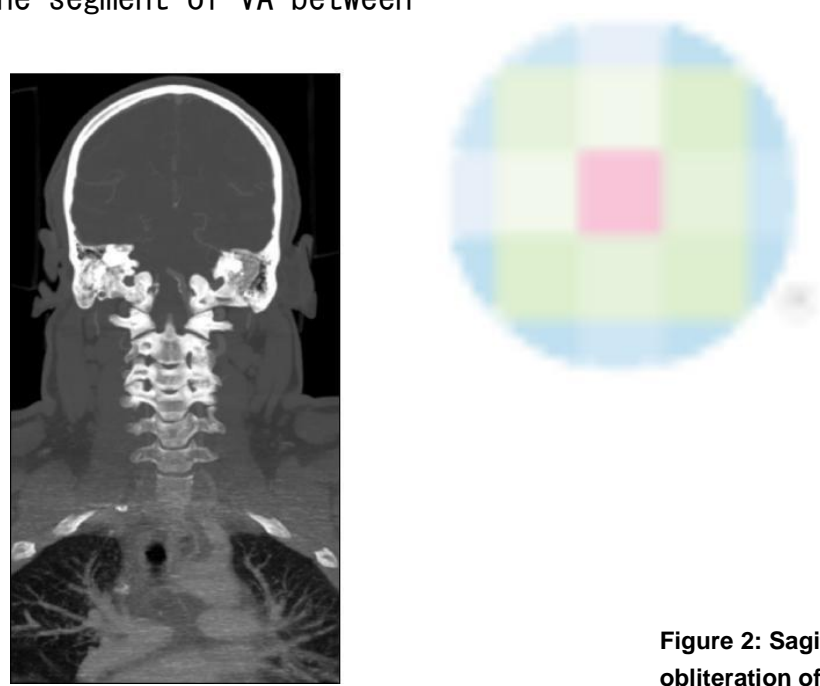

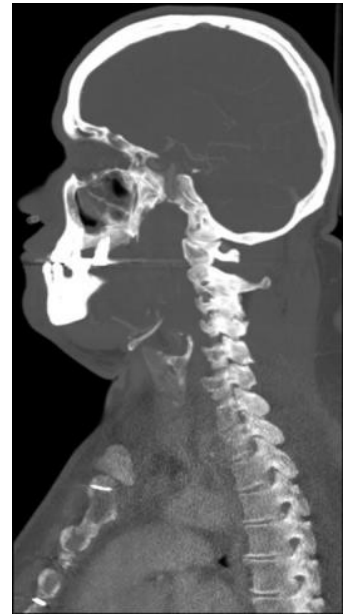

Figure 2: Sagittal computerized tomography revealing C2-C3 fusion and obliteration of $\mathrm{C} 3-\mathrm{C} 4$ transverse foramen

C1 and C2 being most susceptible to rotational occlusion. [2] As the head turns around a fixed atlantoaxial joint, the axis on the contralateral side is anteriorly shifted and can result in stenosis or occlusion of the VA. [3] In this scenario, vertebrobasilar insufficiency was very likely to occur given the presence of the patient' $s$ hypoplastic right VA.

Developmental and degenerative variations can predispose one to Bow Hunter's syndrome. Depth of the vascular groove, ossification or hypertrophy of the atlantooccipital membrane, spondylotic osteophytes, facet joint hypertrophy and thickened tendons of longus coli, and anterior scalenus muscles all contribute to VA stenosis. [4] The resultant vertebrobasilar insufficiency associated with Bow Hunter's syndrome manifests itself via symptoms of localized ischemia including vertigo, diplopia, dysarthria, dysphagia, drop attacks, and/or nausea. [5]

Conservative options are available for the treatment of Bow Hunter's syndrome and involve behavioral modifications, restrictive braces, cervical traction, as well as the use of
Figure 1: Coronal computerized tomography revealing C2-C3 fusion and obliteration of $\mathrm{C} 3-\mathrm{C} 4$ transverse foramen 


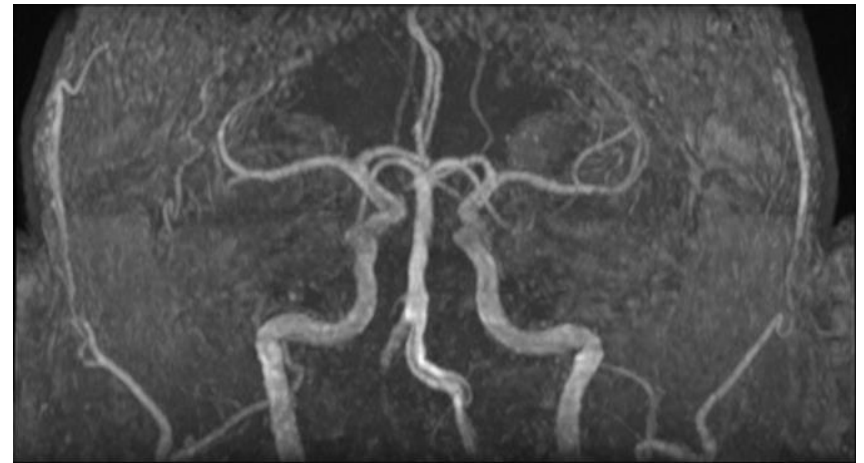

Figure 3: Magnetic resonance imaging of brain showing hypoplastic right vertebral artery

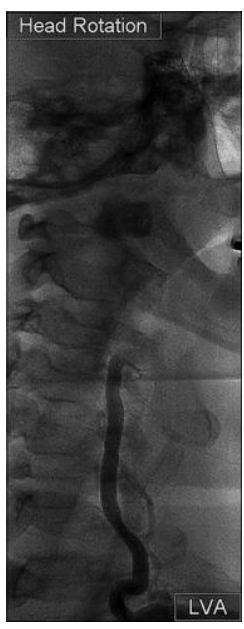

Figure 4: Dynamic fluoroscopy of the left vertebral artery showing obliteration of the left vertebral artery flow with head rotation to the left Asian Journal of Neurosurgery | Volume 13 | Issue 1 | January-March 2018 
Cai, et al:: Bow Hunter's syndrome

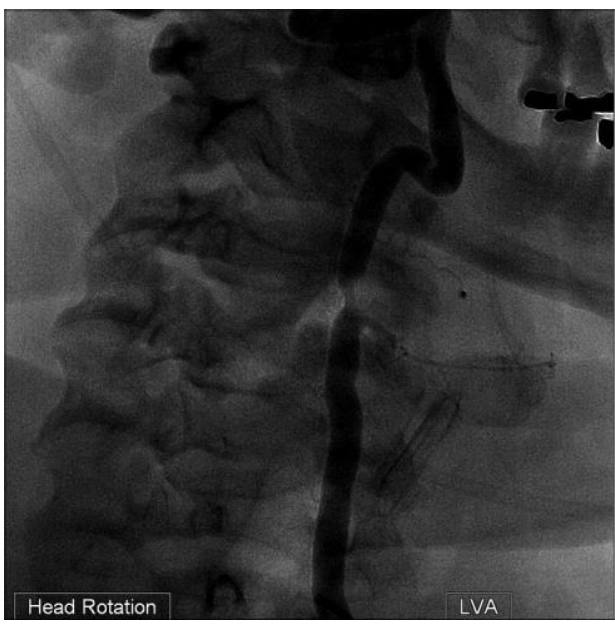

Figure 5: Dynamic fluoroscopy of the left vertebral artery showing reconstitution of the left vertebral artery flow with head in neutral position

anticoagulant medications. When conservative therapy fails to provide relief, surgical procedures may be utilized. Decompression and fixation are two techniques available for the treatment of Bow Hunter's syndrome. Fixation is directed at removing rotational ability at the affected joint. A 50-70\% reduction in head rotation is associated with fusions at the atlantoaxial joint. Subaxial fusions result in minimal reductions in head rotation. Decompressive procedures are effective at stabilizing blood flow while minimizing the reduction in head rotation. [6] Factors such as the surgeon's preference, presence of anterior foraminal stenosis due to uncinate osteophytes, or the presence of posterior foraminal stenosis due to a hypertrophied joint facet should be considered when deciding upon a certain technique. VA anomalies should also be considered prior to surgery. In a prior study, we reported the number of interarterial anomalies to be $10 \%$. We are reporting a case where the patient had a hypoplastic anomaly of the right $V A$ and a dynamically compressible left VA, a presentation that has not previously been described in the literature.

\section{Conclusion}

The symptoms associated with vertebrobasilar insufficiency as manifested in Bow Hunter' $s$ syndrome range from asymptomatic to life-threatening. Patients who are successfully treated with medical therapy including

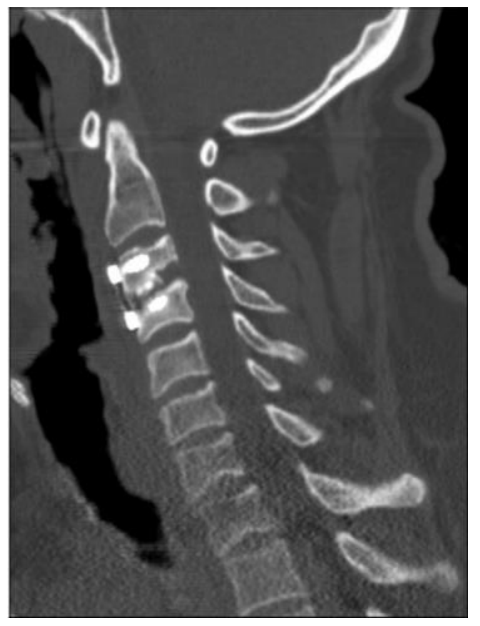

Figure 6: Sagittal computerized tomography after anterior cervical discectomy and fusion at $\mathrm{C} 3-\mathrm{C} 4$

anticoagulants may remain symptom-free for undetermined periods of time; however, the underlying pathology behind the symptoms can only be corrected with surgical treatment. Accurate anatomical knowledge is of the utmost 
importance for not only determining the extent of the occlusion but also in the planning of surgical management. In addition, the use of dynamic fluoroscopy for both the initial assessment and postoperative follow-up proves to be a valuable tool in the management of Bow Hunter's syndrome.

Financial support and sponsorship

Nil.

Conflicts of interest

There are no conflicts of interest.

\section{References}

1. Choi KD, Choi JH, Kim JS, Kim HJ, Kim MJ, Lee TH, et al. Rotational vertebral artery occlusion: Mechanisms and long-term outcome. Stroke 2013;44:1817-24.
2. Bulsara KR, Velez DA, Villavicencio A. Rotational vertebral artery insufficiency resulting from cervical spondylosis: Case report and review of the Iiterature. Surg Neurol 2006;65:625-7.

3. Husni EA, Bell HS, Storer J. Mechanical occlusion of the vertebral artery. A new clinical concept. JAMA $1966 ; 196: 475-8$.

4. Seki T, Hida K, Akino M, Iwasaki Y. Anterior decompression of the atlantoaxial vertebral artery to treat bow hunter' s stroke: Technical case report. Neurosurgery $2001 ; 49: 1474-6$.

5. Shimizu T, Waga S, Kojima T, Niwa S. Decompression of the vertebral artery for bow-hunter's stroke. Case report. J Neurosurg 1988;69:127-31.

6. Sullivan HG, Harbison JW, Vines FS, Becker D. Embolic posterior cerebral artery occlusion secondary to spondylitic vertebral artery compression. Case report. J Neurosurg $1975 ; 43: 618-22$. 